\title{
XXVI. Some electromagnetic effects related to the Hall effect
}

\section{E.P. Adams}

To cite this article: E.P. Adams (1914) XXVI. Some electromagnetic effects related to the Hall effect, Philosophical Magazine Series 6, 27:158, 244-252, DOI: $10.1080 / 14786440208635085$

To link to this article: http://dx.doi.org/10.1080/14786440208635085

曲 Published online: 08 Apr 2009.

Submit your article to this journal $\pi$

Џll Article views: 2

Q View related articles $\square$

Citing articles: 4 View citing articles 5 
3. In the case of elements such as platinum, mercury, and lead there is a small amount of an "extra-radiation" with an absorption coefficient of about $40 \mathrm{~cm}^{-1}$ in lead. This "extra-radiation" may be characteristic of the radiator.

4. The production of secondary $\gamma$ rays plays an important part in the absorption of the primary $\gamma$ rays.

5. Lead produces more secondary $\gamma$ rays than does an equal mass of aluminium, zine, or tin.

6. The distribution of the secondary radiation has been examined under various conditions.

I wish to express my best thanks to Prof. Rutherford for the supply of large quantities of emanation and for his advice during the course of these experiments.

XXVI. Some Electromagnetic Fffects related to the Hall Iffect. By E. P. Adams, Professor of Physics, Princeton University *.

A NUMBER of interesting electromagnetic effects A were described by Professor ('orbino two years ago (Physikalische Zeitschrift, xii. pp. 561, 842, 1911), which have an important bearing on the electron theory of metallic conduction. The theory of the experiments given by him does not lead directly to results which admit of numerical calculation. He introduces both positive and negative carriers of electricity, and his resnlts involve the mobility of these two kinds of ions. It is the purpose of this paper to develop the theory of these effects in terms of the electrons and their characteristics. While it is certain that the simple electron theory of metallic conduction, involving collisions with the atoms like those between hard elastic spheres, is very imperfect, it seems worth while to examine its consequences in these cases. The results obtained are very simple and furnish an excellent opportunity of testing the hypotheses involved.

The three electromagnetic effects predicted and discovered by Professor Corbino are the following :-

(1) A metallic circular disk in which a uniform radial electric current flows is placed in a magnetic field at right angles to its plane. There results a circular current in the plane of the disk such that the current density is inversely proportional to the radius.

(z) The disk with the radial current flowing in it is * Communicated by the Author. 
suspended in a magnetic field so that its normal is inclined to the lines of force. A torque is produced tending to turn the disk.

(3) A radial current is induced in the disk on exciting a magnetic field at right angles to its plane. An equal and opposite radial current is induced when the field is destroyed.

It is necessary to consider the motion of electrons in a circular disk when a radial current flows. Let $\mathrm{O}$ be the whole radiul current, $\tau$ the specific resistance of the metal, $d$ the thickness of the disc, $r_{1}$ and $r_{2}$ its internal and external radii. The current must be thought of as entering the disk through a wire of radius $r_{1}$ at its centre, and as leaving uniformly from all points of its periphery of radius $r_{2}$.

The radial force acting on an electron of charge $e$ is

$$
\frac{\mathrm{C} \tau}{2 \pi r d}=\frac{a}{r} \quad \text { where } a=\frac{\mathrm{C} \tau}{\mathrm{\Sigma} \pi d}
$$

and there is no tangential force. The equations of motion of an electron are:

$$
\begin{aligned}
& \frac{d^{2} r}{d t^{2}}-r\left(\frac{d \theta}{d t}\right)^{2}=\frac{a \dot{e}}{m r}, \quad . \quad . \quad . \quad . \quad . \\
& \frac{1}{r} \frac{d}{d t}\left(r^{2} \frac{d \theta}{d t}\right)=0, \quad . \quad . \quad . \quad . \quad . .
\end{aligned}
$$

$m$ is the mass of the electron. (2) gives

$$
r^{2} \frac{d \theta}{a t}=k, . \quad . \quad \cdot \quad \cdot \quad \cdot . .
$$

and this with (1) gives

$$
\frac{d^{2} r}{d t^{2}}=\frac{k^{2}}{r^{3}}+\frac{a e}{m r} .
$$

Integrating :

$$
\left(\frac{d r}{d t}\right)^{2}=\mathrm{A}-\frac{k^{2}}{r^{2}}+\frac{2 a e}{m} \log r . . . \quad . \quad .
$$

The electrons are assumed to move equally in all directions with velocity $v$ between collisions when there is no electric field. The effect of the electric field is to give them a drift in its direction. After a collision the electron begins to move with velocity $v$ in a wholly random direction. During its free path, until its next collision, it is acted upon by the electric force; we must now find the arerage velocity it 
acquires in this direction. Consider an electron which moves away from a collision at the point $r^{\prime} \theta^{\prime}$, in a direction making an angle $\alpha$ with the radius. At the beginning of its free path its radial and tangential velocities are:

$$
\begin{gathered}
\frac{d r}{d t}=v \cos \alpha \\
r \frac{d \theta}{d t}=v \sin \alpha .
\end{gathered}
$$

From (3) the constant $k$ is determined :

$$
k=r^{\prime} v \sin \alpha,
$$

and using this in (4) the constant $A$ is determined:

$$
\mathrm{A}=v^{2}-\frac{2 a e}{m} \log r^{\prime}
$$

Therefore the radial velocity of the electron during its free path is given by

$$
\left(\frac{d r}{d t}\right)=v^{2} \cos ^{2} \alpha+\frac{2 a e}{m} \log \frac{r}{r^{\prime}} .
$$

At the end of its free path the value of $r$ will be approximately

$$
r^{\prime}+\lambda \cos \alpha,
$$

where $\lambda$ is the length of the free path of the electron. So its radial velocity at the end of its free path is given by :

$$
\left(\frac{d r}{d t}\right)^{2}=v^{2} \cos ^{2} \alpha+\frac{2 a e}{m} \log \left(1+\frac{\lambda \cos \alpha}{r^{\prime}}\right)
$$

$\frac{\lambda \cos \alpha}{r^{\prime}}$ ! is very small compared with unity. This may then be replaced by :

$$
\left(\frac{d r}{d t}\right)^{2}=v^{2} \cos ^{2} \alpha+\frac{2 \alpha e \lambda \cos \alpha}{m r^{\prime}}
$$

The average radial velocity acquired under the infuence of the electric force is one-half the difference of its radial velocities at the end and beginning of its free path. This gives for the radial velocity during the free path:

$$
\overline{d r}=\frac{1}{2} v \cos \alpha\left\{\left(1+\frac{2 a e \lambda}{m v^{2} r^{\prime} \cdot \cos \alpha}\right)^{\frac{1}{2}}-1\right\} \text {. }
$$


As the second term in the parentheses is very small compared with unity, this reduces to

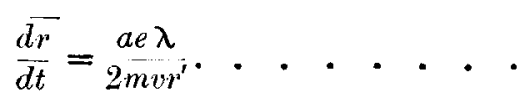

Let there be $\mathrm{N}$ free electrons in unit volume of the motal. Then the radial current in the disk is

$$
\mathrm{C}=\frac{\pi\left(a e^{2} \lambda \mathrm{N}\right.}{m v}
$$

Substituting the value of $a$, we git

$$
\tau=\frac{2 m v}{e^{2} \lambda \mathrm{N}} \text {. }
$$

Let $\mathrm{T}$ be the free time of an electron so that $\lambda=v \mathrm{~T}$. The specific resistance of the metal is then given by

$$
\boldsymbol{\tau}=\frac{2 m}{e^{2} \mathrm{~T} N} \text {. . . • • • • }
$$

This is the same expression for the specific resistance which is obtained by considering the flow of electrons along: a straight bar.

Now suppose that the disk, carrying the radial current C, is placed in a uniform magnetic field of intensity $\mathrm{H}$, at right angles to the plane of the disk. Let the directions of $r, \theta$, $\mathrm{H}$ form a right-handed system.

The equations of motion of an electron are :

$$
\begin{aligned}
m\left\{\frac{d^{2} r}{d t^{2}}-r\left(\frac{d \theta}{d t}\right)^{2}\right\} & =\frac{a e}{r}+\mathrm{H} e r \frac{d \theta}{d t}, \\
\frac{m}{r} \frac{d}{d t}\left(r^{2} \frac{d \theta}{d t}\right) & =-\mathrm{H} e \frac{d r}{d t},
\end{aligned}
$$

where, of course, $e$ must be taken as negative for the electrons. In the second of these equations for $\frac{d r}{d t}$ we may put the average velocity acquired by the electron under the electric force alone, since the effect of the magnetic field is small. This average radial velocity is given by (5).

We thus find :

$$
r^{2} \frac{d \theta}{d t}=-\frac{1}{2} \frac{\mathrm{H} e^{2} \mathrm{~T} a d}{m^{2}}+\mathrm{B} .
$$

At the beginning of a free path, when $t=0, r \frac{d \theta}{d t}=0$ on 
the average since the electromagnetic force has not had time to act on the electron. So $B=0$. At the end of a free path $t=\mathrm{T}$. Thus the average tangential velocity acquired by an electron during its free path is

$$
r \frac{d \bar{\theta}}{d t}=-\frac{\mathrm{H} e^{2 r} T^{2} a}{4 m^{2} r} .
$$

The whole circular flowing is :

$$
\mathrm{I}=-\int_{r_{1}}^{r_{2}} \frac{\mathrm{HN} e^{3 \mathrm{~T}^{2}} a d}{4 m^{2}}-\frac{d r}{r}=-\frac{\mathrm{H} e \mathrm{TC}}{4 \pi m} \log \frac{r_{2}}{r_{1}} . .
$$

This current changes in sign with the radial current $\mathrm{C}$. Since $e$ is negative, the current $\mathrm{I}$ is positive if $\mathrm{C}$ is directed outwards from the centre and negative if directed inwards. In the former case the circular current in the disk has the same direction as the magnetizing current, and in the latter case it has the opposite sign.

'This circular current was measured by Professor Corbino in a bismuth disk by determining the current induced in a concentric coil of wire outside the disk when the radial current was made or broken. The direction of the current was found to agree with that given by this view of the effect. In order to obtain a uniform radial current a copper wire which passed through a hole in one pole-piece of the electromagnet was fastened to the centre of the disk. A copper ring was joined to the periphery of the disk and to this ring a circular copper plate was joined parallel to the disk. A copper tube enclosing and insulated from the central wire was joined to the centre of the copper plate. This method made it certain that the current introduced into the disk was wholly radial. Nothing is said in bis paper of making a radial slit in the circular copper plate. Unless this were done, the effect of the circular current in the bismuth plate would bo partly balanced by the effect of the opposite current in the copper plate. He states that when the bismuth plate was replaced by a copper plate no effect was observed. This is just the result to be expected if no radial slit to prevent a circular current were made.

If we assume, however, that the whole effect obtained was the result of the circular current in the bismuth plate, this experiment gives a simple method of calculating the free time of an electron in bismuth. For a radial current $\mathrm{C}=15$ amperes in a magnetic field of 3700 , the circular current I was found to be 0.26 ampere. The radius $r_{2}$ of the disk was $3.25 \mathrm{~cm}$. The radius $r_{1}$ of the copper wire is not given, 
but it is reasonable to suppose that it was such that $\frac{r_{2}}{r_{1}}=15$. Using these values in eq. (7) with $\frac{e}{m}=10^{7}$ we find

$$
\mathrm{T}=2 \cdot 10^{-12} \text {. }
$$

The specific resistance of bismuth is

$$
\begin{aligned}
& \tau=1 \cdot 310^{4}, \\
& e=1 \cdot 610^{-20} .
\end{aligned}
$$

Eq. (6) thus gives for the number of free electrons in unit volume of bismuth :

$$
\mathrm{N}=5.10^{20} .
$$

It was found that the circular current was not proportional to the magnetic field, but increased less rapidly than the magnetic force. But there are reasons (Phys. Review, xxiv. p. 428, 1907) for believing that the free time of an electron is dependent upon the magnetic force, and this may account for the differences observed between theory and experiment.

Professor Corbino regards this effect of the magnetic field upon the current in the disk as something distinct from the Hall effect. It is certainly true that if the Hall effect is defined as a bending of the equipotential lines by the magnetic field, then this effect is different. For in this case the equipotential lines are concentric circles hoth in and out of the magnetic field. It seems to me, however, that the effect described by Professor Corbino is, essentially, the Hall effect, but measured in a different way from that usually employed. In fact, his method seems to be the ideally simplest way to measure the Hall effect. In the ordinary method, where a uniform current is sent through a rectangular plate, and the transverse current is led out at two points, complications in the current distribution in the plate are introduced which make it difficult to apply the electron theory of metallic conduction. Experiments are now in progress here to measure the effect in a number of metals in addition to bismuth.

The view that Professor Corbino's effect is really the Hall effect receives support when we examine the inverse case. Suppose a circular current flows throagh a disk in a magnetic field. A radial current should result. In order to introduce a circular current into the disk the arrangement 
shown in the accompanying figure might be adopted. A sector of small angle is cut out of the disk and two wires of

Fig. 1.

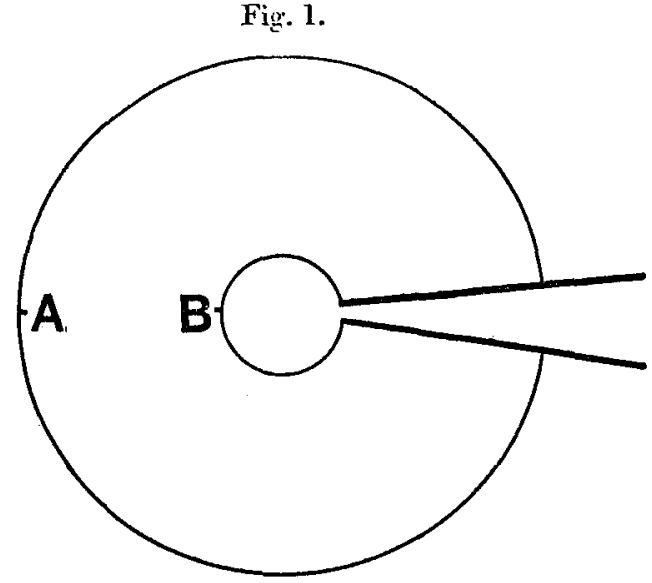

very low resistance soldered to the edges. If the current is introduced through these wires, then, if their resistance can be neglected, it may be shown that the circular current density in the disk is inversely proportional to the radius; there is no radial current. The equipotential lines are the radii. Now apply a magnetic field perpendicular to the disk. Obviously it is impossible to lead out the radial current so as to make this experiment the exact inverse of the former one. The best that can be done is to connect the extremities, say $A, B$, of a radius to a galvanometer which will show that these points are no longer at the same potential. But this effect is certainly the Hall effect. The rectangular plate ordinarily employed has only to be thought of as bent and stretched into the form shown.

For the theory of the two other electromagnetic effects, we shall follow Professor Corbino's argument, but expressed in terms of the characteristics of the electrons. Suppose the disk carrying the radial current $\mathrm{C}$ is suspended in a uniform magnetic field so that its normal makes an angle $\phi$ with the lines of force. The energy of the disk in the magnetic field is given by

$$
\mathrm{W}=-\frac{1}{2} \int_{r_{1}}^{r_{2}} \frac{\mathrm{HN} e^{3} \mathrm{~T}^{3} a d}{4 m^{2} r} \pi r^{2} d r \mathrm{H} \cos ^{2} \phi,
$$

assuming the magnetic permeability to be unity. 
Hence

$$
\mathrm{W}=-\frac{e \mathrm{H}^{2} \mathrm{CTS}}{16 \pi m} \cos ^{2} \phi,
$$

where $\mathbf{S}$ is the effective area of the disk.

Thus the torque on the disk is

$$
-\frac{\partial W}{\partial \phi}=-\frac{e \mathrm{H}^{2}(\mathrm{TS}}{16 \pi m} \sin 2 \phi .
$$

The existence of this torque was shown, but not measured, by Corbino in the case of bismuth. Its measurement would furnish another means of calculating the free time of an electron.

The third effect, the induction of a radial current in the disk on exciting a normal magnetic field, is also easily explained. At any instant during the rise of the magnetic field let $E$ be the radial electromotive force and $e$ the radial current. Then

$$
\frac{d \mathrm{~W}}{d t}=\mathrm{E} c=-\frac{e c \mathrm{~S}}{16 \pi m} \frac{d}{d t}\left(\mathrm{H}^{2} \mathrm{~T}\right) .
$$

We assume that $\mathrm{T}$ is a function of the magnetic force. 'Therefore :

$$
\mathrm{E}=-\frac{e \mathrm{~S}}{16 \pi m} \frac{d}{d t}\left(\mathrm{H}^{2} \mathrm{~T}\right)
$$

The resistance of the disk is

$$
\mathrm{R}=\frac{T}{2 \pi} d \log \frac{r_{2}}{r_{1}}
$$

Hence the whole quantity of electricity that flows radially during the setting up of the magnetic field is :

$$
\mathrm{Q}=-\frac{e \mathrm{~S}}{16 \pi m \mathrm{R}} \mathrm{H}^{2} \mathrm{~T} \text {. }
$$

On comparison with the theory of these effects given by Professor Corbino, we see that his "differential moment of the ions," $\mathrm{E}^{\prime \prime}$, is to be replaced by

$$
\frac{1}{2} \frac{e^{\prime} \mathrm{T}}{m} \text {. }
$$

For the field strength 3700, Professor Corbino determined 
this by experiment from the third effect to be $1.5 \pm 10^{-5 *}$. Hence

$$
\mathrm{T}=3 \cdot 10^{-12},
$$

which is in good agreement with the value $\mathrm{T}=2 \cdot 10^{-12}$ found from the first effect.

XXVII. Analysis of the $\gamma$ Rays from the Uranium Products. By H. Richardson, M.Sc., Beyer Fellow, University of Manchester $†$.

T $N$ previous papers $\ddagger$ it has been shown that the $\gamma$ rays 1 emitted by the radium, thorium, and actinium products can be analysed into groups of different penetrating powers. All the radioactive substances which emit $\beta$ rays have been examined except the uranium products, viz. uranium $X_{1}$ and uranium $\mathrm{X}_{2}$. In order to complete the series the analysis of the $\gamma$ rays from these substances has been undertaken, and confirmatory evidence concerning the atomic weight of actinium has been obtained.

The uranium $X$ used in the experiments was separated from 3 kilograms of pure uranium nitrate. The method of separation employed was that used by Soddy and Russell $§$ in their early investigations on the penetrating power of the $\gamma$ rays from uranium $X$. It consists in the fractional crystallization of the uranium nitrate from a solution of dencity $2 \cdot 06$. Under these conditions, the uranium nitrate which crystallizes out only carries with it about one-seventh of the total amount of uranium $X$ present, and the remaining six-sevenths may be poured off with the mother liquor. By repeating this process about three-quarters of the uraninm $X$ can be obtained in a solution which contains only a few grams of uranium nitrate. The uranium $X$ was finally separated by precipitation with ammonia and anmonium carbonate in the presence of ferric chloride. By this means the uranium $X$ was obtained together with a small amount of ferric hydroxide, and the whole source thus consisted of

* The value given by Professor Corbino for $\mathrm{E}$ is $7 \cdot 7.10^{-5}$. This is to be multiplied by 2 on account of an error corrected in the second of his papers referred to ; and it should be divided by 10 since in another place he has used the ampere as the unit of current instead of the absolute unit.

+ Communicated by Prof. F. Rutherford, F.R.S.

\pm Rutherford and Richardson, Phil. Mag. xxv. p. 722 (1913), xxvi. p. $324(1913)$.

\$ Soddy and Russell, Phil. Mag. xriii. p. 620 (1909). 